\title{
EDITORIAL
}

cell biology

\section{A prize for protein degradation}

Selective protein degradation by the ubiquitin-proteasome pathway is a fundamental regulatory strategy that fulfils essential roles in various cellular processes. In recognition of the central importance of this pathway to cell physiology, this year's Nobel prize for Chemistry has been awarded to Avram Hershko, Aaron Ciechanover and Irwin Rose for their discovery of ubiquitin-mediated protein degradation.

In the late 1970s, in the course of studying an ATP-dependent proteolytic system in reticulocytes, Hershko and Ciechanover identified a heat-stable polypeptide that they named ATP-dependent proteolytic factor 1 . APF-1 was later shown to be identical to ubiquitin, a protein so named for its ubiquitous expression, by Keith Wilkinson and colleagues. After purifying APF-1, Hershko, Ciechanover and Rose went on to make the remarkable discovery that APF-1 could be covalently attached to degradation substrates, and that this attachment seemed to be important for protein degradation by an ATP-dependent pathway. Over the next few years, a series of ground-breaking biochemical studies from the trio established the fundamental sequence of events leading to protein ubiquitination, and also identified the essential factors necessary for ubiquitination. Other individual contributions, in addition to their Nobel citation, include biochemical identification of the prototype of a now extensive family of deubiquitinating enzymes and identification of the cyclosome (APC/C), a cell-cycle-regulated factor required for cyclin ubiquitination and mitotic exit.

A successful partnership of complementary approaches has been instrumental in driving the pace of discovery in many areas of cell biology, and protein degradation is no exception. Complementing the biochemical studies in reticulocyte lysates, analysis of the pathway by Alexander Varshavsky and colleagues in both mammalian tissue culture cells and budding yeast led to the identification and characterization of multiple components of the ubiquitin-proteasome pathway and established the functional relevance of this pathway in multiple cellular processes. These studies also led to a molecular dissection of the first degradation signal, the $\mathrm{N}$-degron, and thus helped uncover the basis of substrate selectivity in the ubiquitin pathway.

The field has come a long way since the time when protein degradation was consigned to the distinctly unfashionable task of clearing the cell of garbage proteins. The emphasis of molecular biology back then was squarely on understanding gene expression as a regulatory mechanism. Although the ubiquitin pathway certainly functions to dispose of aberrant proteins, it is now abundantly clear that a high degree of substrate selectivity is built into the system. Indeed, as more factors that regulate substrate specificity are being identified, the regulatory potential of ubiquitination might eventually be found to even outstrip that of phosphorylation. As the repertoire of functions for ubiquitin has expanded into non-proteolytic roles and the ubiquitin family has diversified to include ubiquitin-related proteins, ubiquitin seems to have lived up to its name - ubiquitous not only in expression but also in function.
Focusing on disease

For most cell biologists, day-to-day motivation is probably derived from the desire to understand the molecular mechanisms that control basic cellular functions. At the same time, most researchers will probably agree that bridging the gap between basic science and medicine is an important eventual goal of research in cell biology. Recent successes in high-profile projects such as the human genome project, combined with a decade long emphasis on the translational promise of basic research, have increasingly mobilized the general public, and their governmental representatives in particular, to ask for palpable therapeutic returns from publicly funded research.

The mechanistic understanding of disease per se is not the central focus of the majority of papers published in Nature Cell Biology. This is, of course, to be expected, as the main editorial premise of this journal is the understanding of basic cellular functions irrespective of whether the mechanism under study is known to be defective in disease. Nonetheless, we do agree that medical progress is a major goal of cell biological research, and we think it is important to occasionally take a step back and assess how our understanding of cell biology may translate to medicine. This is exactly our aim with this somewhat expanded issue of Nature Cell Biology, which presents a collection of review articles focusing on the cell biology of disease.

Of course, it would be impossible to cover the cell biology of disease comprehensively within one issue; instead, our approach has been to highlight a few areas of cell biology in which recent research has made progress from a disease or therapeutic perspective, and which are also representative of research across the editorial scope of the journal. Our hope is that by initiating a discussion of the molecular mechanisms of disease using the language of cell biology, we can encourage further cross-fertilization between disease and basic research.

What emerges as an underlying theme across all the articles in this focus issue is that the flow of information, ideas and inspiration is decidedly two-way. Research aimed at understanding the mechanisms of disease, particularly through human genetics and mouse knockouts, has yielded a wealth of information about the genes involved in specific cellular functions. This is particularly apparent in the review by Thomas Jentsch and colleagues describing how specific functional roles for the vastly complex molecular diversity of ion channels has mainly been identified by characterizing mutants in human disease or through gene targeting in mice. The Perspective by Philippe Halban describes a perhaps more traditional route where excitement over the possibilities of cell-replacement therapies for diabetes has fuelled huge interest and research into the source of cells normally used in vivo to replace pancreatic $\beta$ cells.

Good examples of how disease research has informed basic research into molecular mechanisms, and of course the reverse, can be found in each of the articles presented here. Thus, the cross-fertilization between disease and basic research seems to be alive and well. Cell biology is an obvious perspective from which to approach dysfunction in disease, and with the exponentially expanding knowledge from genomics, proteomics and bioinformatics, now may truly be the time for a unified effort to understand the cell biology of disease. 